\title{
Commentary
}

\section{Could there be a Synthesis between Western and Oriental Medicine, and with Sasang Constitutional Medicine in Particular?}

\section{Denis Noble}

\author{
Department of Physiology, Anatomy and Genetics, Oxford University, Parks Road, Oxford OX1 3PT, UK
}

\begin{abstract}
Attitudes towards oriental medicine are changing for two major reasons. The first is that many patients, even in the West, are choosing to use its practitioners and methods. The second is that the rise of Systems Biology may offer a better basis for dialogue, and even for synthesis, between the oriental and Western traditions. However, a lot of work is needed to clear the way for such dialogue and synthesis. Much of this work should be devoted to clarifying the meanings of the terms used, and the framework of theory and practice within which oriental methods operate. But it is also necessary for Systems Biology itself to mature as a discipline, particularly at the higher levels of biological organization since it is at these levels that oriental medicine derives its ideas and practice. Higher level Systems Biology could be a basis for interpretation of the Korean version of oriental medicine: Sasang constitutional medicine since it seeks patient specific analysis and treatment, and the mathematical methods of systems biology could be used to analyze the central concept of balance in Sasang.
\end{abstract}

Keywords: oriental medicine - Physiome Project - Sasang constitutional medicine - Systems Biology

\section{Introduction}

Many of the problems in understanding between different medical traditions arise from language and translation. I start this commentary, therefore, with some definitions and clarifications. I will refer to the empirical sciencebased medical practice developed in the West over the last two centuries as Western medicine. Of course, it is now practiced around the world and much of the scientific research underlying it occurs today in the East as well as in the West. Similarly, traditional oriental medicine (TOM) in its various forms is now practiced worldwide, but it is still convenient to characterize it by its oriental origins. These definitions are straightforward and widely understood. Unfortunately, that does not apply to the terms and methods used in the two traditions. It is a fundamental mistake to translate the terms used in oriental

For reprints and all correspondence: Prof. Denis Noble, CBE, FRS, Department of Physiology, Anatomy and Genetics Parks Road, Oxford OX1 3PT, UK. Tel: +44-1865-272528; Fax: +44-1865-272554;

E-mail: denis.noble@dpag.ox.ac.uk medicine as simple references to identifiable components within the anatomy and physiology of Western medicine [see for example, the comments in Kim and Pham (1) on the meanings of the Chinese characters usually translated as spleen, pancreas, kidney, lung and liver, and the earlier and ground-breaking work of Porkert (2)]. I will return to these translation problems later.

Until recently, the two traditions have been viewed by many as incompatible alternatives. But two current trends in medical science and practice have made it important to reconsider the relationship between Western medical science and TOM in its various forms. We may eventually need a synthesis of the two approaches. Since this issue of the journal is devoted to Sasang constitutional medicine (SCM), the article ends with specific reference to this form.

\section{Patient Choice}

The first is a trend in practice. What in the West we refer to as complementary and alternative medicine (CAM) 
has seen a very significant growth in practice, as more patients are choosing to go to practitioners of these methods. As Kim and Pham (1) note in their article, this trend has also been seen in oriental countries. Naturally, within Korea, this has focused on the uniquely Korean form of TOM, SCM (four types) (3,4). In Japan, Kampo (the Japanese version of TOM) is now taught in many medical schools (5-7). Similar trends have been seen in China.

There may be multiple causes for this trend in practice; however, high amongst these causes must be frustration with the fact that, while the reductive scientific approach has seen spectacular advances in treating what we might, misleadingly, call simple diseases (i.e. ones with simple causes, even if the disease itself is complicated), there has been a perception that we are failing to advance as rapidly on diseases with multiple causes. These notably include cardiovascular disease, metabolic disease, nervous and psychological diseases and cancer. And even diseases with a simple cause, like HIV, are proving difficult to treat.

Actually, this perception is not fully justified. Cancer was once seen as a death sentence. In many cases it can now be successfully treated. Similarly, significant progress has occurred in the treatment of cardiovascular disease. But these developments have failed to keep up with a major change in population profile. In developed countries, this has shifted towards longevity. As people reach ages at which multiple-cause diseases are more likely, the number of cases of age-related disease inevitably rises. This simple statistical fact explains the paradox that while great advances have been made, many more patients are presenting with these complex diseases. We succeed spectacularly in keeping people alive through the ages at which death from infectious diseases was formerly prevalent, to see them arrive years later in the clinics as patients with multifactorial diseases. To the patient, this might seem like a failure when, instead of a simple drug, like an antibiotic, curing their disease, he or she finds a more nuanced reaction involving multiple tests and more tentative, even experimental, forms of treatment. It is not surprising that patients then turn to other forms of medical practice. This can be characterized as the negative reason for turning to CAM or TOM. They are seen as alternatives to the failures of Western medicine.

I suspect though that there is also a positive reason. People may well be choosing CAM or TOM because they prefer its philosophical and social basis; it is perceived to pay more attention to the patient. The major successes of Western medicine have been reductive in approach: to discover the low-level cause of the disease and treat it. If the low-level cause is an invading organism or a lack of a nutrient, or hormone, or too much of a particular chemical, then the treatment often works. The problem is that this encourages treatment of the disease while ignoring the particular patient who has the disease. The approach is not, of course, intended to be dehumanizing but, in many contexts where time and money are short, it does in fact lead to such a perception, even becoming the undeniable reality. This is the kind of situation that was immortalized in the best-selling novel, The House of God (8), which initially scandalized many medical practitioners in America for its irreverent uncovering of shocking treatment of, and attitudes towards, elderly patients, but which has now become standard reading in many medical schools and the subject of serious articles and books on the ethics of medicine $(9,10)$. Incidentally, the real Samuel Shem was a doctoral student in my Oxford laboratory before practicing medicine in the USA. I have watched this particular argument in Western medicine at close hand and with unusual interest.

This argument applies with particular force to SCM since it specifically characterizes the patient first, by assigning a category, and then deciding on treatment. It can therefore be seen as an early form of patientspecific medicine. Later in this article I will ask the question what it must do to remain so.

\section{The Metaphorical Nature of Current Ideas on Genes and Organisms}

The dehumanizing philosophy also lies deep inside the metaphorical language widely used to convey the results of Western medical and biological science to the general public. The hugely popular book, The Selfish Gene, illustrates the problem. The organism, the person, is reduced to a merely transient vehicle for the 'real' enduring entities, its genes, which 'created us, body and mind' (11). And in case that message was not fully absorbed, Richard Dawkins re-iterated it with even more emphasis in The Extended Phenotype: '[Readers] should imbibe the fundamental truth that an organism is a tool of DNA rather than the other way round' (12). Later, highly significant, qualifications, such as 'Genes aren't us' (13) have failed to redress the damage. We now have a culture that looks for 'genes for everything' and which hailed the sequencing of the human genome as 'reading the book of life'.

There is in fact no justification in medical and biological science for any of these metaphors. The test for this is simple. Choose alternative, integrative rather than reductive, metaphors and ask the question 'what empirical evidence could there be that one or the other is correct?' As I show in my book, The Music of Life (14), there are no such empirical tests. This is not science, although it masquerades as science and is widely perceived to be so. Therein lies the problem. We have been seriously misled. So much, so that when the Human Genome Project reached its first complete drafts in 2000 the publicity 
surrounding this phenomenal technical and scientific achievement promised that reading 'the book of life' would see new cures for disease naturally emerge from reading the 'book'; cures literally tumbling out with hundreds of new identified targets for therapy. The reality however is that even fewer new drugs are coming to market and at ever greater cost. There is something wrong therefore with the way in which these scientific achievements have been presented.

\section{Systems Biology}

This leads me to the second relevant trend, which is the development of what is now called Systems Biology. Identifying the individual genes and proteins that are found in living systems can be viewed as breaking the system down into its ultimate components. That is why we characterize this approach as reductionist. But can the organism actually be reduced to these components? Or is this not rather like taking a hammer to a computer and breaking it up into its millions of transistors and wires? Looking at those components arrayed on a laboratory bench, would we know how the computer worked? The answer is obviously 'no'. We would need the blueprint for putting it all back together again. We would also need the software called the operating system, and all the various programs that make it function. One of the hopes of the genome project was that, within the sequences of DNA we would find the 'program of life' to use Monod and Jacob's colorful metaphor (15). But what we actually find is more like a database, a set of templates for the construction of proteins and a set of switches that need to be turned on or off to make the system work.

It turns out that crucial information for interpreting the database lies within the rest of the cell, not just in the DNA sequences. I have recently reviewed this question in some depth (16). Genes, interpreted narrowly as sequences of DNA, need the fertilized egg cell to be interpreted, just as a virus cannot operate and reproduce outside a living cell. And later in development, interaction with the rest of the organism and with the environment is critical in determining the nature of the organism. Organisms are interaction machines not Turing machines blindly following a step-by-step program.

Even the architects of the Human Genome Project say exactly this. In his fascinating biography, Craig Venter writes 'One of the most profound discoveries I have made in all my research is that you cannot define a human life or any life based on DNA alone...... Why? Because 'An organism's environment is ultimately as unique as its genetic code' (17). Precisely so and, one should add, the environment is an open system.

John Sulston is also cautious: 'The complexity of control, overlaid by the unique experience of each individual, means that we must continue to treat every human as unique and special, and not imagine that we can predict the course of a human life other than in broad terms' (18).

Sequencing the human genome has therefore brought us right up against the problem of complexity in biological systems. This is the challenge that 21 st century biology faces. Its foundations must therefore be built on how to integrate our knowledge, rather than simply follow a reductive mode. Having broken life down into its molecular components, the greater problem is going to be how to put those components back together again and to understand the logic of life at all the various biological levels. This raises difficult questions. Building is far more difficult than breaking up. This is the domain of Systems Biology. It is not an entirely new discipline. In fact I would identify its origins as being, first, with the 19th century French physiologist Claude Bernard (19), who introduced the idea of control of what he called the internal environment, and thus the concept of homeostasis (20). It can also be seen as originating in the early applications of mathematics to biological systems, notably the analysis of the nerve impulse by Hodgkin and Huxley (21). Complexity absolutely requires mathematics since, beyond a certain number of interactions between components, intuition (guesswork) fails us. We need to calculate the expected behavior of a system. Systems Biology has therefore been greatly advanced by the development of fast, powerful computers (22).

Although we can identify these historical roots, Systems Biology has really taken off only recently (23-28). It is therefore a relatively young initiative and it is far too early to judge how successful it may be in avoiding and resolving the difficulties of the reductionist approach. Nevertheless, there are encouraging signs in the analysis of new drugs developed within the pharmaceutical industry (29). Significantly, some of these are drugs with multiple actions, an approach that can also be seen to be important in herbal medicine, which forms a major part of oriental medicine. This approach is also leading to the development of what are called virtual organs and systems: reconstruction of the function of whole organs and systems as part of a project called the Human Physiome Project $(30,31)$. The European version of this is actually called the Virtual Physiological Human.

The really significant philosophical difference between Systems Biology and reductionist biology is that Systems Biology focuses on processes rather than components. It is in this sense more holistic, though I have reservations about the word 'holistic' in this context [see also (32)]. Systems Biology, it seems to me, is rather a multilevel analysis of biological processes $(20,30)$. It is certainly not simply a top-down analysis, as the word 'holistic' may suggest. It may respect holistic approaches more than does reductionist biology, but it is not itself strictly a holistic method. For related reasons, it is not 
just a study of emergent properties: properties that are inherent in the set of components, but which only appear when they interact. It was Sydney Brenner who coined the phrase 'middle-out' to emphasize that the approach is neither bottom-up nor top-down. Function at higher levels in organisms depends on an interaction between the genome, the cells, tissues and organs, and the environment, with the higher levels and the environment acting as constraints on the processes at lower levels. Without those constraints life could not exist. There is no privileged level of causality $(16,20)$.

\section{Conditions for Dialogue and Synthesis}

Could Systems Biology form the basis for some kind of dialogue at least, and perhaps even a synthesis, between the Western and oriental traditions, as suggested for example by Jane Qiu (33)? This was the subject of a meeting held in Oxford recently at which scientists from both traditions were present. There was general agreement that System Biology, and particularly the existence of 'downward causation' (i.e. higher level constraint of lower level processes), does open up the possibility of constructive dialogue between the Western and oriental medical traditions in a way that was not possible within the dominant reductive mode of biology.

The reasons for this agreement were:

1. The characterization of forms of 'downward causation', including both feedback and constraint, could be a way of linking the two traditions.

2. Systems Biology can identify multiple actions as being more beneficial than single site actions [e.g. multiple action drugs within the Western medical tradition, most recently ranolazine-see (29), and the use of synergistic drug combinations (34)]. This could open the way to a better understanding of, and development of, herbal medicine, since this also depends on synergistic actions of multiple components.

3. Systems Biology recognizes the importance of control of the genome by higher levels (via epigenetic marking and control) including even the role of behavioral and social factors. This can be seen, for example, in the work of Weaver et al. (35-37) on epigenetic inheritance of stroking behavior in rats. This form of downward causation spans all the levels of biological organization and could open the way to dialogue on the central role of the mind in oriental Medicine.

4. Korean Sasang can be viewed as a patient specific form of treatment. Genomics and Systems Biology are also looking towards the development of patient specific medication and treatment. However, Systems Biology is not looking towards ancient Galenic or other interpretations of constitution.
The question therefore arises whether oriental medicine could be open to investigation on this question? I think it can. Kim et al. (38) chart the way forward in applying genomics to SCM.

5. Could oriental medicine become consistent with the need for regulation and clinical trial evidence? There are already examples of successful clinical trials of acupuncture for pain relief and of meditation therapy for depression. Oriental medicine should not be fearful of clinical trials, though it has to be admitted that there are special difficulties in establishing effective controls in such trials. The correct placebo for a treatment like acupuncture is much more difficult to arrange than is a sugary pill!

6. But perhaps, as noted in my introduction, the biggest problem is historical and cultural. In the West, oriental medicine is thought by many people to include many kinds of 'magic', mysterious effects that are perceived as anti-scientific. This raises the question whether oriental medicine could be de-mythologized?

What do I mean by demythologizing? I mean whether it would be possible to map concepts like 気 $(k i, q i)$, that at first sight closely resemble that of 'vital energy', to systems-level concepts that are empirically testable and do not simply resurrect the old concept of vital energy, a concept that was specifically rejected by scientists like Claude Bernard. There are similar questions with concepts such as 精 (jing essence?), 神 (shen spirit?), 陰 (yin), 陽 (yang), 脉 (mai vessel), and as Kim and Pham show (1), for the various Chinese characters for organ systems. My proposal is that we might more profitably identify these with systems processes not as separate substances. In this context, it is interesting that in a recent study of the practice of Traditional Chinese Medicine in modern China, Hsu (39) ends with the conclusion:

It was the process which brought about disharmony, not the material aspects.... this conception of the body in its disordered state was common to qigong and Chinese medicine

\section{Conclusions}

I suggest that dialogue and synthesis will need to take place in several stages:

(a) Translation and interpretation. The outlines for some of this work are already clear $(1,2,39-42)$. A good example of the approach required is to be found in $\mathrm{Lu}$ and Needham's study of acupuncture and moxibustion, relating the historical oriental texts to interpretations using modern biology (43). There is a solid base, therefore, on which to 
build. But much more work will be required. It is not as simple as producing a better dictionary of oriental medical terms. Those terms acquire their meanings within a complete semantic frame, in turn determined by historical and sociological backgrounds that need to be understood. Moreover, translation and interpretation depends not only on understanding the source language, but also on the target language into which the interpretation is done. An important conclusion of this article is that the language of Systems Biology is still developing. Since we do not yet know the higher-level concepts that will form its foundations in the future, we cannot yet know what would be the best way to map oriental medical concepts onto those of Systems Biology. The processes of translation and interpretation are therefore on-going tasks, working within the frameworks of the two cultures involved, each of which is developing.

(b) An essential component of future work will therefore be the development of Systems Biology in a direction that enables understanding of physiological and pathological processes at the higher levels of organization. We are a long way from achieving this at the current time. The greater effort and funding in Systems Biology is at the lower levels of gene-protein networks. The Physiome Project attempts to redress the balance here, but it also is a long way from integrating together organ systems that could correspond, for example, to those postulated by SCM and TOM in general.

(c) The application of Systems Biology to oriental medicine. Systems Biology is a highly quantitative discipline. Some even define it in terms of the ways in which mathematics is applied to biology. In addition to molecular biology, genomics, proteomics and bioinformatics, it also has roots in biological engineering and in mathematical biology. It is nothing less than a revolution in biology, bringing to it the same rigor as mathematics has brought to physics, engineering and chemistry. oriental medicine, by contrast, is not mathematical, though concepts of balance of the kind discussed in some of the articles in this volume could obviously be expressed mathematically.

I suspect therefore that, in addition to changing the nature of modern biological science, Systems Biology may eventually also change the nature, or at least the characterization of TOM. This conclusion is particularly relevant to the tradition of SCM. It could examine its fundamental basis in two ways. The first, as outlined by Kim et al. (35), is that the concept of constitutional types could be re-examined via genomic studies.
The main challenge here will be that the concepts of constitutional type at the phenotype level (as in Sasang) and at the genomic level are different since there is no simple relation between genotype and phenotype. The causal links are complex (15). But Sasang scientists are in a good position to do this work since they have access to patients who are naturally classified according to phenotype constitutional group. Relating these classifications to genotypes would be a very important first step. The second is that the concept of balance between systems could be treated mathematically, just as Claude Bernard $(18,19)$ envisioned the mathematical analysis of his concept of homeostasis, the processes that maintain the constancy (via various forms of balance) of the internal environment. As the Physiome Project advances, such an application to the balance concept in Sasang could become possible.

\section{References}

1. Kim J-Y, Pham D-D. SCM as holistic tailored medicine. eCAM 2009.

2. Porkert M. The difficult task of blending Chinese and Western science: the case of modern interpretations of traditional Chinese medicine. In: Li GH (ed). Explorations in the History of Science and Technology in China. Shanghai: Zhonghua wenshi luncong, 1982, 553-72.

3. Ahn K-S. The Essence of Oriental Medicine. Seoul: Sonamoo Publishing Co., 1999.

4. Song I-B. An Introduction to Sasang Constitutional Medicine. Paju: Jimoondang, 2005.

5. Kosoto Hiroshi. History of Kampo. Chinese and Japanese tradition of herbal medicine (in Japanese). Tokyo: Taishukan, 1999.

6. Japan Society for Oriental Medicine. Introduction to Kampo (in Japanese). Nankodo, 2002.

7. Japan Society for Oriental Medicine. Introduction to Kampo. Tokyo: Elsevier Japan, 2005.

8. Shem S. The House of God. New York: Dell, 1978, 2003.

9. Kohn M, Donley C (eds). Return to the House of God. Kent, $\mathrm{OH}$ : Kent State University Press, 2008.

10. Noble D. The birth of the house of god. In: Kohn M, Donley C (eds). Return to The House of God: Medical Resident Education, 1978-2008. Kent, Ohio, USA: Kent State University Press, 2008, $1-8$.

11. Dawkins R. The Selfish Gene. Oxford: Oxford University Press, 1976.

12. Dawkins R. The Extended Phenotype. London: Freeman, 1982.

13. Dawkins R. A Devil's Chaplain. London: Weidenfeld and Nicolson, 2003.

14. Noble D. The Music of Life. Oxford: Oxford University Press, 2006.

15. Monod J, Jacob F. Teleonomic mechanisms in cellular metabolism, growth and differentiation. Cold Spring Harbor Symp Quant Biol 1961;26:389-401.

16. Noble D. Genes and causation. Philos Trans Royal Soc A 2008;366: 3001-15.

17. Venter C. A Life Decoded. London: Penguin Books, 2007.

18. Sulston J, Ferry G. The Common Thread. London: Bantam Press, 2002.

19. Bernard C. Introduction à l'étude de la Médecine Expérimentale. Paris: Flammarion, 1865, 1984 (reprint).

20. Noble D. Claude Bernard, the first Systems Biologist, and the future of Physiology. Exp Physiol 2008;93:16-26.

21. Hodgkin AL, Huxley AF. A quantitative description of membrane current and its application to conduction and excitation in nerve. J Physiol 1952;117:500-44.

22. Noble D. The rise of computational biology. Nat Rev Molecular Cell Biol 2002;3:460-3. 
23. Kitano H. Systems biology: a brief overview. Science 2002;295: $1662-4$.

24. Palsson BO. The challenges of in silico biology. Nat Biotechnol 2000; $18: 1147-50$.

25. Facciotti MT, Marc T, Bonneau R, Hood L, Baliga NS. Systems Biology experimental design - considerations for building predictive gene regulatory network models for prokaryotic systems. Curr Genom 2004;5:527-44.

26. Bray D. Wetware. A Computer in Every Cell. New Haven, CT: Yale University Press, 2009.

27. Marcus FM. Bioinformatics and Systems Biology: Collaborative Research and Resources. New York, NY USA: Springer, 2008.

28. Kohl P, Noble D. Systems biology and the virtual physiological human. Mol Syst Biol 2009, Published online: 28 July 2009, doi: $10.1038 / \mathrm{msb} .2009 .51$.

29. Noble D. Computational models of the heart and their use in assessing the actions of drugs. J Pharmacol Sci 2008;107:107-17.

30. Bassingthwaighte JB, Hunter PJ, Noble D. The Cardiac Physiome: perspectives for the future. Exp Physiol 2009;94:597-605.

31. Hunter PJ, Crampin EJ, Nielsen PMF. Bioinformatics and the physiome project. Brief Bioinform 2008;9:333-43.

32. Kupiec J-J. The Origin of Individuals: A Darwinian Approach to Developmental Biology. London: World Scientific Publishing Company, 2009.

33. Qiu J. Traditional medicine. A culture in the balance. Nature 2007;448:126-8

34. Lehar J, Krueger AS, Avery W, Heilbut AM, Johansen LM, Price ER, et al. Synergistic drug combinations tend to improve therapeutically relevant selectivity. Nat Biotechnol 2009; 27:659-66.

35. Weaver ICG, D'Alessio AC, Brown SE, Hellstrom IC, Dymov S, Sharma $\mathrm{S}$, et al. The transcription factor nerve growth factorinducible protein A mediates epigenetic programming: altering epigenetic marks by immediate-early genes. J Neurosci 2007;27: 1756-68.

36. Weaver ICG. Life at the interface between a dynamic environment and a fixed genome. In: Janigro D (ed). Mammalian Brain Development. Amsterdam: Humana-Elsevier, 2009, in press.

37. Weaver ICG, Cervoni N, Champagne FA, D'Alessio AC, Sharma S, Sekl JR, et al. Epigenetic programming by maternal behavior. Nat Neurosci 2004;7:847-54.

38. Kim B-y, et al. Genetics approach to elucidation of Sasang constitutional medicine. eCAM 2009.

39. Hsu E. The Transmission of Chinese Medicine. Cambridge: Cambridge University Press, 1999.

40. Hsu E (ed). Innovation in Chinese Medicine. Cambridge University Press: Cambridge, 2001.

41. Porkert M. The Theoretical Foundations of Chinese Medicine: Systems of Correspondence. Cambridge, MA: MIT Press, 1974.

42. Lu GD, Needham J. Medicine in Chinese culture. In: Needham J (ed). Clerks and Craftsmen in China and the West. Cambridge: Cambridge University Press, 1966, 1970, 263-93.

43. Lu GD, Needham J. Celestial Lancets: A History and Rationale of Acupuncture and Moxa. London: Routledge, 2002.

Received March 17, 2009; accepted July 13, 2009 


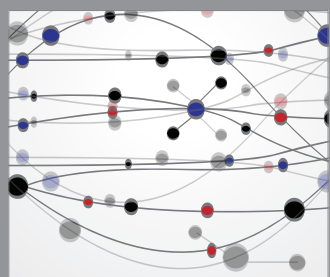

The Scientific World Journal
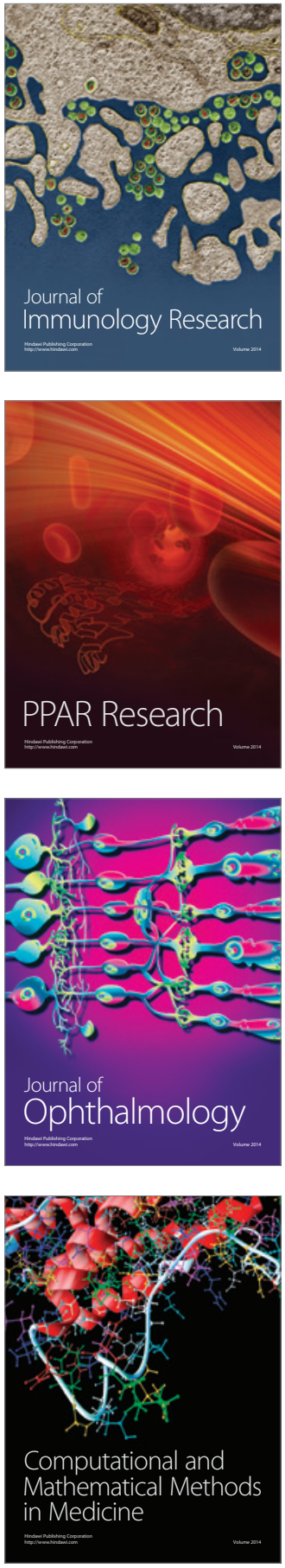

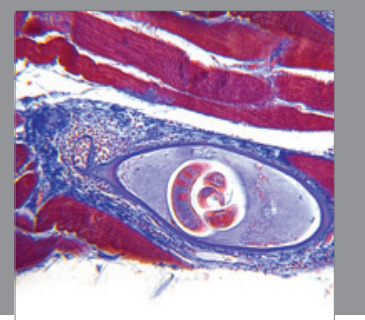

Gastroenterology

Research and Practice
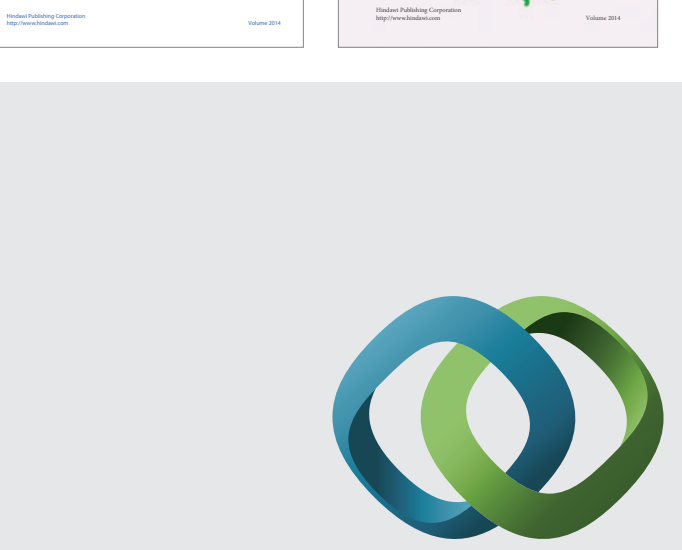

\section{Hindawi}

Submit your manuscripts at

http://www.hindawi.com
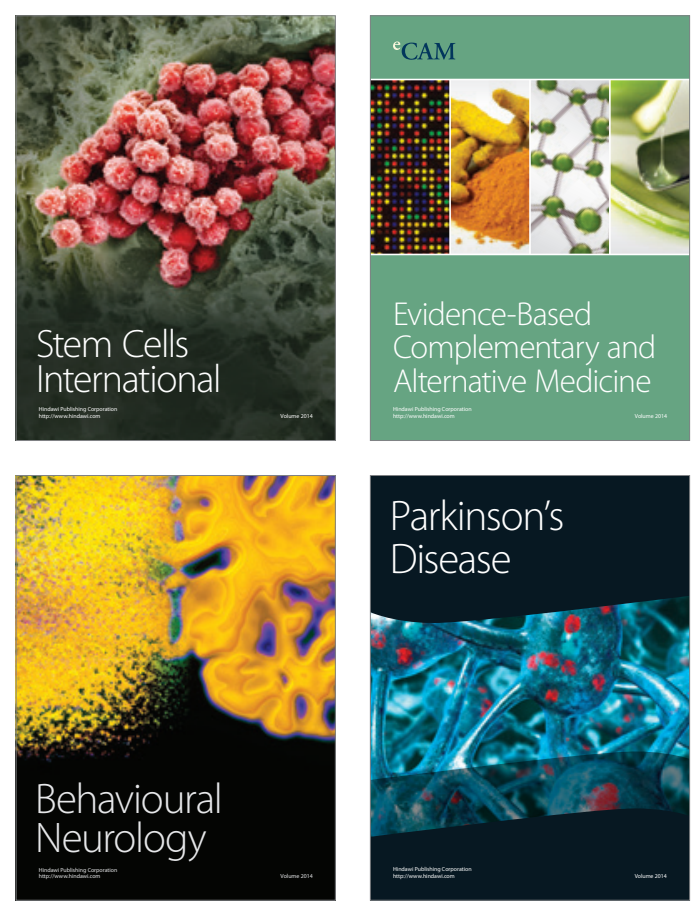

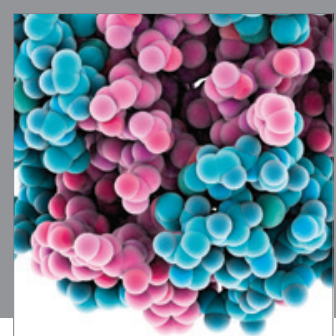

Journal of
Diabetes Research

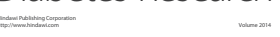

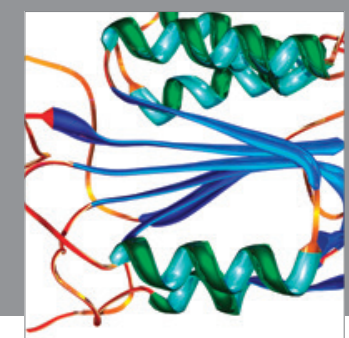

Disease Markers
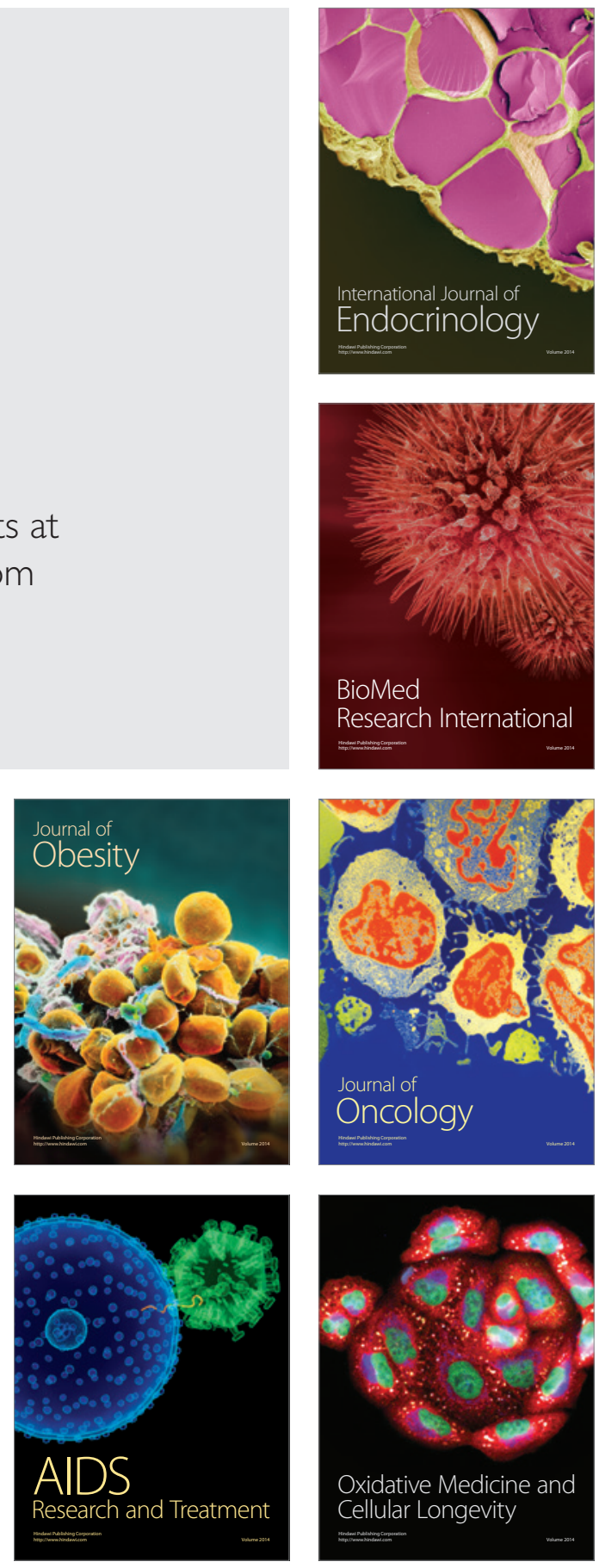